\title{
Convenient synthesis of the pentasaccharide repeating unit corresponding to the cell wall 0 -antigen of Escherichia albertii 04
}

\author{
Tapasi Manna, Arin Gucchait and Anup Kumar Misra*
}

Open Access

\author{
Full Research Paper \\ Address: \\ Bose Institute, Division of Molecular Medicine, P-1/12, C.I.T. Scheme \\ VII M, Kolkata 700054, India \\ Email: \\ Anup Kumar Misra* - akmisra69@gmail.com \\ * Corresponding author \\ Keywords: \\ Escherichia albertii O4; glycosylation; $\mathrm{HClO}_{4} / \mathrm{SiO}_{2}$; O-antigen; \\ pentasaccharide
}

\author{
Beilstein J. Org. Chem. 2020, 16, 106-110. \\ doi:10.3762/bjoc. 16.12 \\ Received: 25 November 2019 \\ Accepted: 15 January 2020 \\ Published: 22 January 2020 \\ Associate Editor: K. N. Allen \\ (c) 2020 Manna et al.; licensee Beilstein-Institut. \\ License and terms: see end of document.
}

\begin{abstract}
A straightforward sequential synthetic strategy has been developed for the synthesis of a pentasaccharide repeating unit corresponding to the cell wall O-antigen of the Escherichia albertii $\mathrm{O} 4$ strain in very good yield with the desired configuration at the glycosidic linkages using thioglycosides and trichloroacetimidate derivatives as glycosyl donors and perchloric acid supported over silica $\left(\mathrm{HClO}_{4} / \mathrm{SiO}_{2}\right)$ as a solid supported protic acid glycosyl activator. The expected configuration at the glycosidic linkages was achieved using a reasonable selection of protecting groups in the manosaccharide intermediates.
\end{abstract}

\section{Introduction}

Diarrheal outbreaks are serious concerns all over the world particularly in the developing countries due to inadequate sanitation systems [1]. In most of the cases, the enteric infections originated due to the intake of less cooked food and contaminated water [2]. Several strains of Shigella [3], Salmonella [4] and enteropathogenic Escherichia coli [5] are commonly known for causing diarrheal infections. Besides the mainstream enteropathogenic bacterium, Escherichia albertii (E. albertii) is an emerging human pathogen causing gastroenteric infections in different countries [6]. Although, this species was identified earlier as Hafnia alvei, later it was redesignated as E. albertii
[7]. E. albertii acted as a causative agent for diarrheal diseases in children with vomiting, fever and abdominal distension [8]. Several strains of E. albertii have been identified till date, which significantly contributed to the spreading of devastating diarrheal infections in different countries [9]. The role of cell wall O-polysaccharides in regulating the virulence properties of bacteria is well established [10]. Recently, Naumenko et al. [11] reported the structure of the repeating unit of the cell wall O-polysaccharide of the E. albertii $\mathrm{O} 4$ strain [11], which is a pentasaccharide comprising of $\alpha$-linked D-galactosamine, $\beta$-linked D-glucosamine, $\beta$-linked D-galactose, $\alpha$-linked L-fucose 
and $\alpha$-linked L-rhamnose moieties. In the recent past, several vaccine candidates have been developed to control bacterial infections by conjugating cell wall polysaccharides with suitable proteins, which include vaccines against Haemophilia influenza type b (Hib) [12,13], meningitis [14], pneumococcal infections [15,16] and enteric diseases such as cholera [17] diarrhea [18] and urinary tract infections [19]. Despite the possibility of isolating the polysaccharides by fermentation techniques, it is difficult to get a significant quantity of polysaccharide fragments from natural sources with adequate purity. Therefore, the development of chemical synthetic strategies is quite pertinent to obtain a requisite quantity of oligosaccharide fragments with adequate purity. In this direction, the total synthesis of the pentasaccharide repeating unit corresponding to the cell wall O-antigenic polysaccharide of the E. albertii $\mathrm{O} 4$ strain using a sequential glycosylation strategy is presented herein (Figure 1).
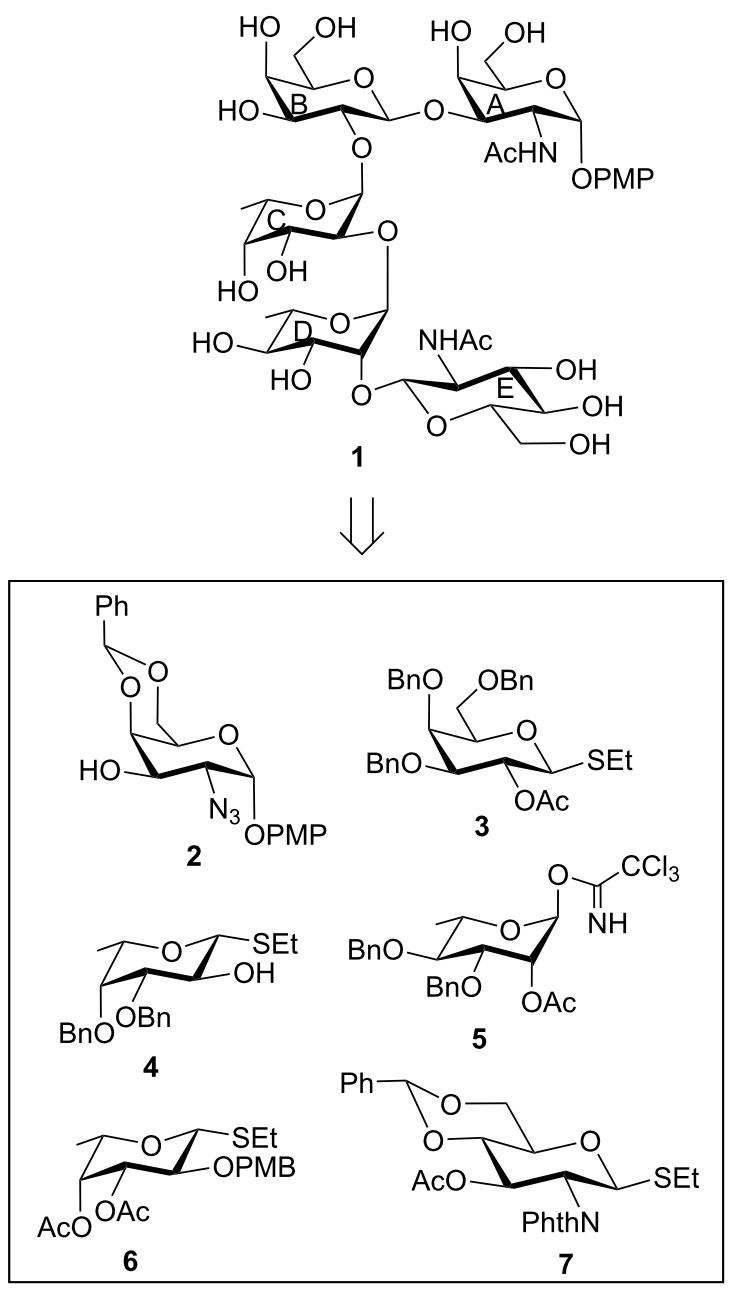

Figure 1: Structure of the pentasaccharide repeating unit corresponding to the cell wall O-antigen of Escherichia albertii $\mathrm{O} 4$ and its synthetic intermediates.

\section{Results and Discussion}

The synthesis of pentasaccharide $\mathbf{1}$ was achieved using a convergent as well as a block synthetic strategy. For this purpose, a series of suitably functionalized monosaccharide intermediates 2 [20], 3 [21], 4 [22], 5 [23], 6 [24] and 7 [25] were prepared from the commercially available reducing sugars utilizing the reaction conditions reported in the literature (Figure 1). Although the monosaccharide intermediates used for the construction of the pentasaccharide derivative $\mathbf{1 5}$ are known in the literature, preparation of these intermediates required multiple step reaction sequences. Having obtained the monosaccharide intermediates, it was decided to proceed through a stepeconomic block synthetic strategy to achieve the target pentasaccharide derivative. Accordingly, stereoselective glycosylation of a D-galactosamine derivative $\mathbf{2}$ with a D-galactose thioglycoside derivative $\mathbf{3}$ in the presence of a combination [26,27] of $\mathrm{N}$-iodosuccinimide (NIS) and perchloric acid supported over silica $\left(\mathrm{HClO}_{4} / \mathrm{SiO}_{2}\right)[28,29]$ furnished disaccharide derivative 8 in $79 \%$ yield, which on de-O-acetylation using sodium methoxide [30] gave the disaccharide acceptor 9 in $95 \%$ yield. NMR spectral analysis of compound $\mathbf{9}$ confirmed its formation with appropriate configuration at the glycosidic linkages [Signals at $\delta 5.54\left(\mathrm{~d}, J=2.5 \mathrm{~Hz}, \mathrm{H}-1_{\mathrm{A}}\right), 5.44(\mathrm{~s}, \mathrm{PhCH})$, $4.54\left(\mathrm{~d}, J=7.5 \mathrm{~Hz}, \mathrm{H}-1_{\mathrm{B}}\right)$ in ${ }^{1} \mathrm{H} \mathrm{NMR}$ and at $\delta 105.2\left(\mathrm{C}-1_{\mathrm{B}}\right)$, 100.6 $(\mathrm{PhCH}), 98.2\left(\mathrm{C}-1_{\mathrm{A}}\right)$ in ${ }^{13} \mathrm{C}$ NMR spectra] (Scheme 1$)$.

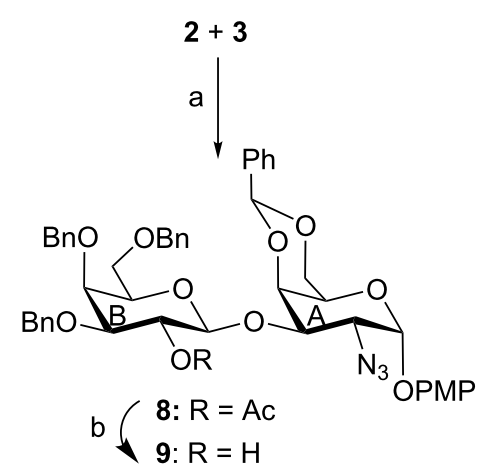

Scheme 1: (a) NIS, $\mathrm{HClO}_{4} / \mathrm{SiO}_{2}$, MS $4 \AA$ A $, \mathrm{CH}_{2} \mathrm{Cl}_{2},-45^{\circ} \mathrm{C}, 1 \mathrm{~h}, 79 \%$; (b) $0.1 \mathrm{M} \mathrm{CH}_{3} \mathrm{ONa}, \mathrm{CH}_{3} \mathrm{OH}$, room temperature, $2 \mathrm{~h}, 95 \%$.

In another experiment, L-rhamnosyl trichloroacetimidate donor 5 was coupled with L-fucosyl thioglycoside acceptor 4 in the presence of $\mathrm{HClO}_{4} / \mathrm{SiO}_{2}$ [31] as activator using an orthogonal glycosylation approach to furnish disaccharide thioglycoside derivative $\mathbf{1 0}$ in $76 \%$ yield, which was directly used in the next level of glycosylation. NMR spectral analysis of compound $\mathbf{1 0}$ unambiguously confirmed its formation [signals at $\delta 5.26(\mathrm{~d}$, $\left.J=1.5 \mathrm{~Hz}, \mathrm{H}-1_{\mathrm{D}}\right), 4.23\left(\mathrm{~d}, J=9.5 \mathrm{~Hz}, \mathrm{H}-1_{\mathrm{C}}\right)$ in ${ }^{1} \mathrm{H} \mathrm{NMR}$ and at $\delta 98.4\left(\mathrm{C}-1_{\mathrm{D}}\right), 84.8\left(\mathrm{C}-1_{\mathrm{C}}\right)$ in ${ }^{13} \mathrm{C}$ NMR spectra] (Scheme 2$)$. It is worth noting that sulfide linkage at the anomeric position 
of compound 4 remained unaffected under the reaction conditions.

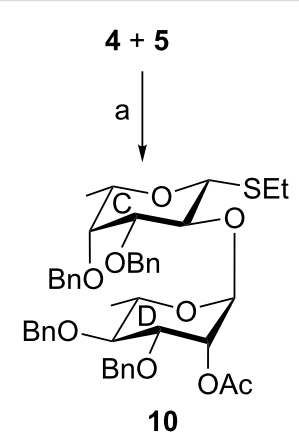

Scheme 2: (a) $\mathrm{HClO}_{4} / \mathrm{SiO}_{2}, \mathrm{CH}_{2} \mathrm{Cl}_{2},-10{ }^{\circ} \mathrm{C}, 1 \mathrm{~h}, 76 \%$.

Having achieved the disaccharide acceptor 9 and the disaccharide thioglycoside donor $\mathbf{1 0}$, a stereoselective glycosylation between them was attempted in the presence of a combination $[26,27]$ of NIS and $\mathrm{HClO}_{4} / \mathrm{SiO}_{2}$ as thiophilic activator. Unfortunately, the required tetrasaccharide derivative $\mathbf{1 1}$ was obtained in a poor yield ( $22 \%$, Scheme 3$)$. It was decided to follow a sequential glycosylation strategy to achieve a significant quantity of compound 11. Accordingly, a stereoselective glycosylation was carried out using compound 9 with L-fucose thioglycoside derivative 6 in the presence of a combination [26,27] of NIS and $\mathrm{HClO}_{4} / \mathrm{SiO}_{2}$ as thiophilic activator. Gratifyingly, the trisaccharide derivative $\mathbf{1 2}$ was obtained in $74 \%$ yield with a newly formed 1,2-cis glycosyl linkage in it. The structural confirmation of compound $\mathbf{1 2}$ was established by its NMR spectral analysis [signals at $\delta 5.67\left(\mathrm{~d}, J=3.0 \mathrm{~Hz}, \mathrm{H}-1_{\mathrm{A}}\right), 5.60$ $\left(\mathrm{d}, J=3.5 \mathrm{~Hz}, \mathrm{H}-1_{\mathrm{C}}\right), 5.50(\mathrm{~s}, \mathrm{PhCH}), 4.79$ (d, $J=7.5 \mathrm{~Hz}$ $\left.\mathrm{H}-1_{\mathrm{B}}\right)$ in ${ }^{1} \mathrm{H} \mathrm{NMR}$ and at $\delta 103.3\left(\mathrm{C}-1_{\mathrm{B}}\right), 100.8(\mathrm{PhCH}), 99.0$ $\left(\mathrm{C}-1_{\mathrm{A}}\right), 97.1\left(\mathrm{C}-1_{\mathrm{C}}\right)$ in ${ }^{13} \mathrm{C}$ NMR spectra]. Compound 12 was subjected to a set of reactions consisting of a one-pot [32] de-Oacetylation and benzylation using benzyl bromide and sodium hydroxide in the presence of tetrabutylammonium bromide (TBAB) followed by oxidative removal [33] of the PMB group using 2,3-dichloro-5,6-dicyano-1,4-benzoquinone (DDQ) to give trisaccharide acceptor $\mathbf{1 3}$ in $72 \%$ yield. Trisaccharide acceptor $\mathbf{1 3}$ was then allowed to couple with L-rhamnosyl trichloroacetimidate donor 5 in the presence of $\mathrm{HClO}_{4} / \mathrm{SiO}_{2}$ as a solid acid activator [31] to provide tetrasaccharide derivative $\mathbf{1 1}$ in $76 \%$ yield, which was de-O-acetylated to furnish tetrasaccharide acceptor $\mathbf{1 4}$ in $94 \%$ yield. The formation of compound $\mathbf{1 1}$ with appropriate configuration at the glycosidic linkages was supported by its NMR spectral analysis [signals at $\delta 5.72(\mathrm{~d}, J=$ $3.5 \mathrm{~Hz}, \mathrm{H}-1_{\mathrm{A}}$ ), 5.58 (d, $\left.J=3.5 \mathrm{~Hz}, \mathrm{H}-1_{\mathrm{C}}\right), 5.51$ (s, $\mathrm{PhCH}$ ), 4.80 (d, $\left.J=7.5 \mathrm{~Hz}, \mathrm{H}-1_{\mathrm{B}}\right), 4.71$ (br s, $\mathrm{H}-1_{\mathrm{D}}$ ) in ${ }^{1} \mathrm{H}$ NMR and at

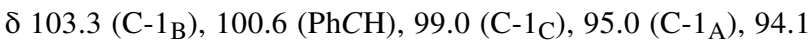
$\left(\mathrm{C}-1_{\mathrm{D}}\right)$ in ${ }^{13} \mathrm{C}$ NMR spectra]. Finally, NIS and $\mathrm{HClO}_{4} / \mathrm{SiO}_{2}-$ promoted stereoselective glycosylation of compound $\mathbf{1 4}$ with D-glucosamine thioglycoside donor 7 furnished the desired pentasaccharide derivative $\mathbf{1 5}$ in $70 \%$ yield. The formation of compound 15 with appropriate configuration at the glycosidic linkages was supported by its NMR spectral analysis [signals at $\delta 5.64\left(\mathrm{~d}, J=3.5 \mathrm{~Hz}, \mathrm{H}-1_{\mathrm{A}}\right), 5.54$ (d, $\left.J=3.0 \mathrm{~Hz}, \mathrm{H}-1_{\mathrm{C}}\right), 5.50$ (d, $\left.J=8.5 \mathrm{~Hz}, \mathrm{H}-1_{\mathrm{E}}\right), 5.48(\mathrm{~s}, \mathrm{PhCH}), 5.41$ (s, $\left.\mathrm{PhCH}\right), 4.96$ (br s, $\left.\mathrm{H}-1_{\mathrm{D}}\right), 4.69\left(\mathrm{~d}, J=8.0 \mathrm{~Hz}, \mathrm{H}-1_{\mathrm{B}}\right)$ in ${ }^{1} \mathrm{H} \mathrm{NMR}$ and at $\delta 103.4$ $\left(\mathrm{C}-1_{\mathrm{B}}\right), 101.6,100.4(2 \mathrm{C}, 2 \mathrm{PhCH}), 100.1\left(\mathrm{C}-1_{\mathrm{E}}\right), 99.0\left(\mathrm{C}-1_{\mathrm{C}}\right)$, $94.9\left(\mathrm{C}-1_{\mathrm{D}}\right), 94.7\left(\mathrm{C}-1_{\mathrm{A}}\right)$ in ${ }^{13} \mathrm{C}$ NMR spectra]. Compound 15 was subjected to a sequence of reactions consisting of (i) reductive transformation of the azido group into an acetamido group by the treatment with thioacetic acid [34]; (ii) transformation of the $N$-phthalimido group into acetamido group using hydrazine hydrate followed by selective N-acetylation [35]; (iii) hydrogenolysis of benzyl ethers and benzylidene acetals over Pearlman's catalyst [36] to furnish the desired pentasaccharide 1 in $49 \%$ overall yield (Scheme 4 ). The structure of compound 1 was unambiguously characterized by its NMR spectral analysis [signals at $\delta 5.37\left(\mathrm{~d}, J=2.0 \mathrm{~Hz}, \mathrm{H}-1_{\mathrm{A}}\right), 5.29(\mathrm{~d}, J=3.5 \mathrm{~Hz}$, $\mathrm{H}-1_{\mathrm{C}}$ ), 5.12 (br s, H-1 $\left.\mathrm{D}\right), 4.73$ (d, $\left.J=7.5 \mathrm{~Hz}, \mathrm{H}-1_{\mathrm{E}}\right), 4.61$ (d, $J=$ $\left.8.0 \mathrm{~Hz}, \mathrm{H}-1_{\mathrm{B}}\right)$ in ${ }^{1} \mathrm{H} \mathrm{NMR}$ and at $\delta 102.2\left(\mathrm{C}-1_{\mathrm{E}}\right), 102.1\left(\mathrm{C}-1_{\mathrm{B}}\right)$, $96.8\left(\mathrm{C}-1_{\mathrm{A}}\right), 96.5\left(\mathrm{C}-1_{\mathrm{C}}\right), 96.0\left(\mathrm{C}-1_{\mathrm{D}}\right)$ in ${ }^{13} \mathrm{C}$ NMR spectra].

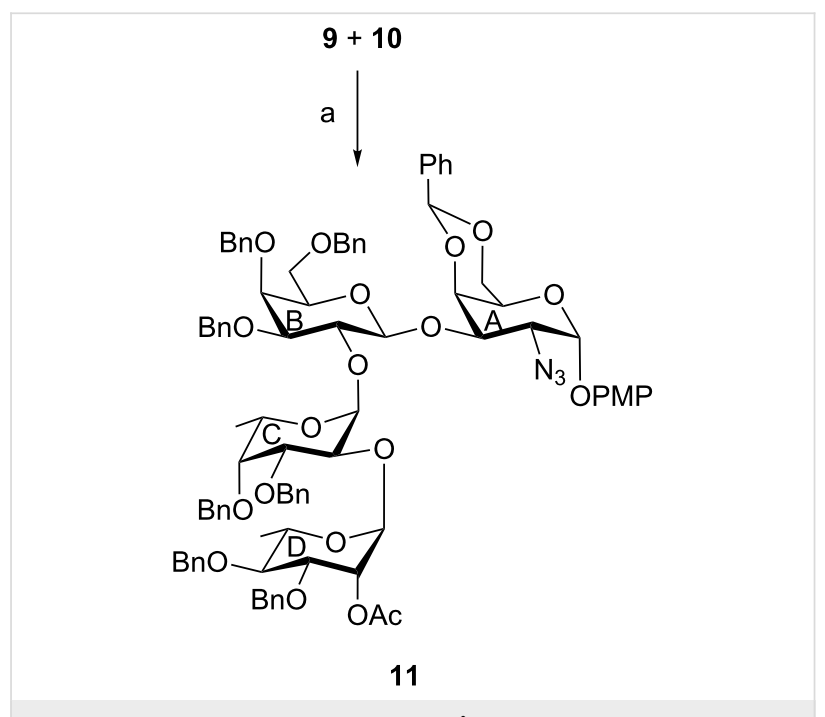

Scheme 3: (a) $\mathrm{NIS}, \mathrm{HClO}_{4} / \mathrm{SiO}_{2}$, MS $4 \AA \mathrm{A}^{-} \mathrm{CH}_{2} \mathrm{Cl}_{2},-40{ }^{\circ} \mathrm{C}, 1 \mathrm{~h}, 22 \%$.

\section{Conclusion}

In summary, a convenient stepwise synthetic strategy has been developed for the synthesis of the pentasaccharide repeating unit of the cell wall O-antigen of Escherichia albertii $\mathrm{O} 4$ in very good yield. Although the target compound can be achieved by block synthetic approach but a better yield of the product was obtained by a sequential approach. $\mathrm{HClO}_{4} / \mathrm{SiO}_{2}$ was used as a solid acid activator in the glycosylation reactions using trichloroacetimidate as well as thioglycoside donors. All interme- 


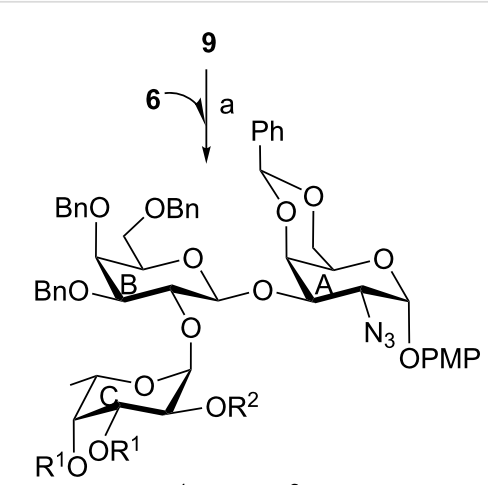

b, $c\left(\begin{array}{l}12: R^{1}=A c ; R^{2}=P M B \\ 13: R^{1}=B n ; R^{2}=H\end{array}\right.$
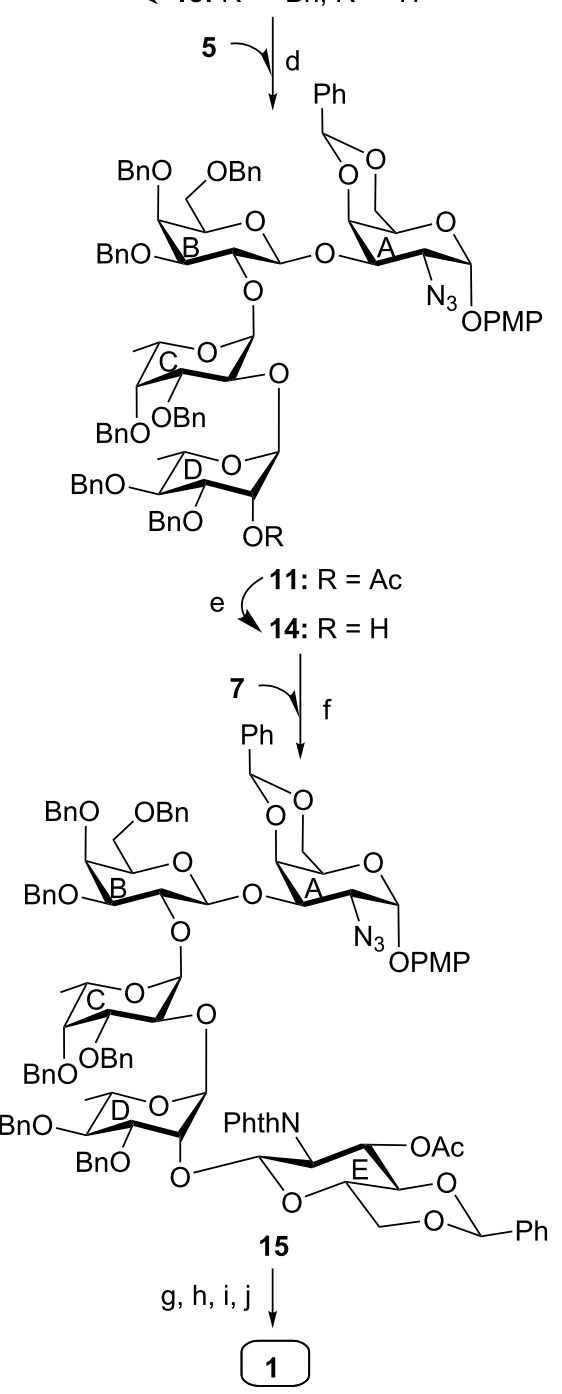

Scheme 4: (a) NIS, $\mathrm{HClO}_{4} / \mathrm{SiO}_{2}$, MS $4 \AA \mathrm{A}^{-} \mathrm{CH}_{2} \mathrm{Cl}_{2},-45{ }^{\circ} \mathrm{C}, 1 \mathrm{~h}, 74 \%$; (b) $\mathrm{BnBr}, \mathrm{NaOH}, \mathrm{TBAB}, \mathrm{THF}$, room temperature, $6 \mathrm{~h}$; (c) DDQ, $\mathrm{CH}_{2} \mathrm{Cl}_{2} / \mathrm{H}_{2} \mathrm{O}(9: 1)$, room temperature, $2 \mathrm{~h}, 72 \%$ in two steps; (d) $\mathrm{HClO}_{4} / \mathrm{SiO}_{2}, \mathrm{CH}_{2} \mathrm{Cl}_{2},-10{ }^{\circ} \mathrm{C}, 1 \mathrm{~h}, 76 \%$; (e) $0.1 \mathrm{M} \mathrm{CH}_{3} \mathrm{ONa}$, $\mathrm{CH}_{3} \mathrm{OH}$, room temperature, $2 \mathrm{~h}, 94 \%$; (f) $\mathrm{NIS}, \mathrm{HClO}_{4} / \mathrm{SiO}_{2}$, MS $4 \AA$, $\mathrm{CH}_{2} \mathrm{Cl}_{2},-15^{\circ} \mathrm{C}, 1 \mathrm{~h}, 70 \%$; (g) $\mathrm{CH}_{3} \mathrm{COSH}$, pyridine, room temperature, $16 \mathrm{~h}$; (h) $\mathrm{NH}_{2} \mathrm{NH}_{2} \cdot \mathrm{H}_{2} \mathrm{O}$, EtOH, $80{ }^{\circ} \mathrm{C}, 12 \mathrm{~h}$; (i) $\mathrm{Ac}_{2} \mathrm{O}, \mathrm{CH}_{3} \mathrm{OH}$, room temperature, $30 \mathrm{~min}$; (j) $\mathrm{H}_{2}, 20 \%-\mathrm{Pd}(\mathrm{OH})_{2} / \mathrm{C}, \mathrm{CH}_{3} \mathrm{OH}$, room temperature, $24 \mathrm{~h}, 49 \%$ in four steps. diate steps were high yielding with excellent stereo outcome in the glycosidic linkages.

\section{Supporting Information}

\section{Supporting Information File 1}

Experimental and analytical data and copies of NMR spectra.

[https://www.beilstein-journals.org/bjoc/content/ supplementary/1860-5397-16-12-S1.pdf]

\section{Funding}

T. M. and A. G. thank UGC and CSIR, New Delhi for providing senior research fellowships. This work was supported by SERB, New Delhi (Project No. EMR/2015/000282 dated 17.09.2015) (AKM).

\section{References}

1. Mokomane, M.; Kasvosve, I.; de Melo, E.; Pernica, J. M.; Goldfarb, D. M. Ther. Adv. Infect. Dis. 2018, 5, 29-43. doi:10.1177/2049936117744429

2. Heredia, N.; García, S. Anim. Nutr. 2018, 4, 250-255. doi:10.1016/j.aninu.2018.04.006

3. Baker, S.; The, H. C. Curr. Opin. Infect. Dis. 2018, 31, 449-454. doi:10.1097/qco.0000000000000475

4. Kariuki, S.; Revathi, G.; Kariuki, N.; Kiiru, J.; Mwituria, J.; Hart, C. A. BMC Microbiol. 2006, 6, 101. doi:10.1186/1471-2180-6-101

5. Nataro, J. P.; Kaper, J. B. Clin. Microbiol. Rev. 1998, 11, 142-201. doi:10.1128/cmr.11.1.142

6. Nimri, L. F. Diagn. Microbiol. Infect. Dis. 2013, 77, 91-95. doi:10.1016/j.diagmicrobio.2013.06.028

7. Huys, G.; Cnockaert, M.; Janda, J. M.; Swings, J. Int. J. Syst. Evol. Microbiol. 2003, 53, 807-810. doi:10.1099/ijs.0.02475-0

8. Sharma, M.; Kniel, K. E.; Derevianko, A.; Ling, J.; Bhagwat, A. A. Appl. Environ. Microbiol. 2007, 73, 4351-4353. doi:10.1128/aem.03001-06

9. Yamamoto, D.; Hernandes, R. T.; Liberatore, A. M. A.; Abe, C. M.; de Souza, R. B.; Romão, F. T.; Sperandio, V.; Koh, I. H.; Gomes, T. A. T. PLoS One 2017, 12, e0171385. doi:10.1371/journal.pone.0171385

10. Silhavy, T. J.; Kahne, D.; Walker, S. Cold Spring Harbor Perspect. Biol. 2010, 2, a000414. doi:10.1101/cshperspect.a000414

11. Naumenko, O. I.; Zheng, H.; Senchenkova, S. N.; Wang, H.; Li, Q.; Shashkov, A. S.; Wang, J.; Knirel, Y. A.; Xiong, Y. Carbohydr. Res. 2017, 449, 17-22. doi:10.1016/j.carres.2017.06.008

12. Zarei, A. E.; Almehdar, H. A.; Redwan, E. M. J. Immunol. Res. 2016, No. 7203587. doi:10.1155/2016/7203587

13. Verez-Bencomo, V.; Fernández-Santana, V.; Hardy, E.; Toledo, M. E.; Rodríguez, M. C.; Heynngnezz, L.; Rodriguez, A.; Baly, A.; Herrera, L.; Izquierdo, M.; Villar, A.; Valdés, Y.; Cosme, K.; Deler, M. L.; Montane, M.; Garcia, E.; Ramos, A.; Aguilar, A.; Medina, E.; Toraño, G.; Sosa, I.; Hernandez, I.; Martínez, R.; Muzachio, A.; Carmenates, A.; Costa, L.; Cardoso, F.; Campa, C.; Diaz, M.; Roy, R. Science 2004, 305, 522-525. doi:10.1126/science.1095209 
14. McCarthy, P. C.; Sharyan, A.; Moghaddam, L. S. Vaccine 2018, 6 , No. 12. doi:10.3390/vaccines6010012

15. Reglinski, M.; Ercoli, G.; Plumptre, C.; Kay, E.; Petersen, F. C.; Paton, J. C.; Wren, B. W.; Brown, J. S. npj Vaccines 2018, 3, No. 53. doi:10.1038/s41541-018-0090-4

16. Kaplonek, P.; Khan, N.; Reppe, K.; Schumann, B.; Emmadi, M.; Lisboa, M. P.; Xu, F.-F.; Calow, A. D. J.; Parameswarappa, S. G.; Witzenrath, M.; Pereira, C. L.; Seeberger, P. H.

Proc. Natl. Acad. Sci. U. S. A. 2018, 115, 13353-13358. doi:10.1073/pnas.1811862115

17. Kay, E.; Cuccui, J.; Wren, B. W. npj Vaccines 2019, 4, No. 16. doi:10.1038/s41541-019-0110-z

18. Berti, F.; Adamo, R. Chem. Soc. Rev. 2018, 47, 9015-9025. doi:10.1039/c8cs00495a

19. Bhatia, S.; Dimde, M.; Haag, R. Med. Chem. Commun. 2014, 5 , 862-878. doi:10.1039/c4md00143e

20. Shit, P.; Gucchait, A.; Misra, A. K. Tetrahedron 2019, 75, 130697. doi:10.1016/j.tet.2019.130697

21. Chernyak, A.; Oscarson, S.; Turek, D. Carbohydr. Res. 2000, 329, 309-316. doi:10.1016/s0008-6215(00)00189-0

22. Sun, J.; Han, X.; Yu, B. Synlett 2005, 437-440. doi:10.1055/s-2004-837221

23. Zhang, J.; Mao, J.; Chen, H.; Cai, M. Tetrahedron: Asymmetry 1994, 5 , 2283-2290. doi:10.1016/s0957-4166(00)86306-5

24. Hendel, J. L.; Wang, J.-W.; Jackson, T. A.; Hardmeier, K.; De Los Santos, R.; Auzanneau, F.-I. J. Org. Chem. 2009, 74, 8321-8331. doi:10.1021/jo901616p

25. Kihlberg, J. O.; Leigh, D. A.; Bundle, D. R. J. Org. Chem. 1990, 55, 2860-2863. doi:10.1021/jo00296a055

26. Mukhopadhyay, B.; Collet, B.; Field, R. A. Tetrahedron Lett. 2005, 46, 5923-5925. doi:10.1016/j.tetlet.2005.06.119

27. Mukherjee, C.; Misra, A. K. Synthesis 2007, 683-692. doi:10.1055/s-2007-965913

28. Chakraborti, A. K.; Gulhane, R. Chem. Commun. 2003, 1896-1897. doi:10.1039/b304178f

29. Chakraborti, A. K.; Gulhane, R. A Process for the Acylation of Various Substrates using a Solid Support Catalyst. Indian Pat. Appl. 266/DEL/2003, March 10, 2003.

30. Zemplén, G. Ber. Dtsch. Chem. Ges. B 1926, 59, 1254-1266. doi:10.1002/cber.19260590626

31. Mukhopadhyay, B.; Maurer, S. V.; Rudolph, N.; van Well, R. M.; Russell, D. A.; Field, R. A. J. Org. Chem. 2005, 70, 9059-9062. doi:10.1021/jo051390g

32. Madhusudan, S. K.; Agnihotri, G.; Negi, D. S.; Misra, A. K. Carbohydr. Res. 2005, 340, 1373-1377. doi:10.1016/j.carres.2005.03.007

33. Oikawa, Y.; Yoshioka, T.; Yonemitsu, O. Tetrahedron Lett. 1982, 23 , 885-888. doi:10.1016/s0040-4039(00)86974-9

34. Shangguan, N.; Katukojvala, S.; Greenberg, R.; Williams, L. J. J. Am. Chem. Soc. 2003, 125, 7754-7755. doi:10.1021/ja0294919

35. Lee, H.-H.; Schwartz, D. A.; Harris, J. F.; Carver, J. P.; Krepinsky, J. J. Can. J. Chem. 1986, 64, 1912-1918. doi:10.1139/v86-315

36. Pearlman, W. M. Tetrahedron Lett. 1967, 8, 1663-1664. doi:10.1016/s0040-4039(00)70335-2

\section{License and Terms}

This is an Open Access article under the terms of the Creative Commons Attribution License

(https://creativecommons.org/licenses/by/4.0). Please note that the reuse, redistribution and reproduction in particular requires that the authors and source are credited.

The license is subject to the Beilstein Journal of Organic Chemistry terms and conditions:

(https://www.beilstein-journals.org/bjoc)

The definitive version of this article is the electronic one which can be found at:

doi:10.3762/bjoc. 16.12 